\title{
Los Géneros Sobre el Pasado en la Vida Mapuche Rural
}

\author{
Genres of the past in Rural Mapuche Life \\ Magnus Course $^{\mathrm{i}}$
}

\begin{abstract}
RESUMEN
Este artículo desafía la amplia asunción relativa a que muchos mapuche rurales de alguna manera carecen de "consciencia histórica", y que esta carencia es un obstáculo para la participación plena en el "movimiento mapuche". En respuesta, busco demostrar la riqueza y diversidad de los involucramientos de los mapuche rurales con el pasado. Empleando los conceptos bakbtinianos de cronotopo y género, sugiero que diferentes formas de encarar el pasado conllevan diferentes asunciones relativas a la persona y la identidad. Presentando material etnográfico sobre canciones personales (ül), sugiero que el pasado es presentado como una serie acumulativa de vidas singulares, y no como un grupo coherente moviéndose a través del tiempo. Luego me aboco al significado del término "Mapuche" como éste es usado en historias personales (nutram), y sugiero que hace referencia tanto a una identidad relacional y productiva como a una comprensión "étnica" de la identidad.
\end{abstract}

Palabras Claves: Mapuche, Canción, Historia, Etnicidad, Identidad.

\begin{abstract}
This article challenges the widely-held assumption that many rural Mapuche people somehow lack "historical consciousness" and that this lack is an obstacle to full participation in the "Mapuche movement." In response, I seek to demonstrate the richness and diversity of rural Mapuche engagements with the past. Utilizing Bakhtin's concepts of chronotope and genre, I suggest that different forms of addressing the past carry different assumptions of person and identity. In presenting ethnographic material on personal songs (ül), I suggest that the past is presented as a cumulative series of singular lives, rather than as a coherent group moving through time. I then turn to the meaning of the term "Mapuche" as used in personal histories (nutram), and suggest that it refers as much to a relational and productive identity, as it does to an 'ethnic' understanding of identity.
\end{abstract}

Key words: Mapuche, Song, History, Ethnicity, Identity.

i Department of Social Anthropology, University of Edinburgh. Chrystal Macmillan Building, 15a George Square. Edimburgo, EH8 9LD, Escocia. E-mail: magnus.course@ed.ac.uk Recibido: Enero 2009. Aceptado: Agosto 2009. 
Mi punto de partida para este artículo se encuentra en la queja frecuentemente articulada por intelectuales mapuche y no mapuche en referencia a que muchos mapuche rurales "no saben nada de su propia historia" o, alternativamente, "no tienen conciencia histórica". Estas acusaciones de ignorancia histórica y la percepción de una suerte de carencia de cualquier cosa que pueda ser denominada como conciencia histórica, son a menudo citadas como la causa central para la relativa falta de participación y entusiasmo de los mapuche rurales en las diversas organizaciones y prácticas comúnmente aglutinadas bajo el nombre de movimiento mapuche. ${ }^{1}$

Ahora bien, aunque en parte estoy de acuerdo con sostener que existe una relación entre la conciencia histórica y el involucramiento con el movimiento mapuche, estoy, por otra parte, en contra de la afirmación relativa a que los mapuche del campo carecen de cualquier tipo de conciencia histórica. En este trabajo pretendo describir la pluralidad y riqueza de representaciones del pasado con que cuenta la vida rural mapuche. A fin de cuentas, tengo la impresión de que el asunto gira en torno al hecho de que la conciencia histórica de la población rural no está ausente, sino más bien es simplemente de una naturaleza diferente a la conciencia sobre la cual se funda predominantemente el movimiento político urbano. Esta discusión no es particularmente original, y lo admito. Se ha expuesto con anterioridad en referencia a muchos otros lugares y contextos. ${ }^{2}$ Sin embargo, estoy lo suficientemente confiado como para afirmar que esta disyunción entre las comprensiones rural y urbana del pasado se yergue como un factor significativo en mucha de la falta de participación en, o de identificación con, el movimiento político mapuche. ${ }^{3}$ Lo que quiero es proveer un breve boceto de la multiplicidad y pluralidad de las representaciones mapuche rurales de las vidas pasadas, y presentar estos géneros de las vidas pasadas no como obstáculos para una participación política plena, sino más bien como fuentes valiosas de inspiración en el proceso de imaginar el pasado mapuche desde una perspectiva propia (cf. Mariman et al 2006).

Comienzo este artículo describiendo un marco analítico que me permite la comparación de diferentes representaciones del pasado sin realizar evaluaciones de ellas en términos de proximidad con una "realidad" histórica objetiva. Empleando una diversidad de ideas extraídas de la teoría histórica y la crítica literaria, exploro como todo género narrativo necesariamente comprende una configuración distinta de la relación entre persona, pasado y sociabilidad. En la segunda parte de este artículo utilizo este marco para describir dos géneros de vidas pasadas sobresalientes en la vida mapuche rural contemporánea: las canciones personales y las narrativas de identidad 
mapuche. Mi opinión a este respecto sería que en estos dos géneros se hace conspicua por su ausencia una versión de la identidad mapuche limitada “étnicamente". La tercera y la última sección abordan cómo esta pluralidad de imaginaciones históricas indígenas se relacionan con la imaginación de una identidad mapuche imperecedera, y limitada étnicamente, a través del tiempo, la versión del pasado sobre la cual se basa gran parte del movimiento político mapuche.

\section{LA IMAGINACIÓN HISTÓRICA}

La aproximación hacia el pasado que tomo en este artículo se fundamenta en la insistencia de Hayden White relativa a que todas las narrativas históricas son sólo eso: narrativas (White 1973, 1987). Comprendido de esta manera, ningún relato singular del pasado puede ser completo: es siempre el resultado de un proceso creativo de selección que conlleva necesariamente tanto inclusión como exclusión.

En los últimos años, el enfoque de White ha sido criticado por centrarse exclusivamente en el lenguaje, a expensas de las maneras en que la memoria histórica puede ser internalizada en prácticas no lingüísticas tales como el ritual, la música o el paisaje. Sin embargo, en mi impresión, estas críticas, al destacar la pluralidad de formas en que el pasado se vuelve presente en las vidas humanas, realmente fortalecen el argumento de White contra la posibilidad de que haya cualquier "verdad" histórica monolítica más allá del reino de la representación humana. Luego, la importancia de las formas no lingüísticas de memoria histórica no niegan el énfasis puesto por White en el hecho de que cualquier representación del pasado es un proceso creativo en conformidad con expectativas culturales.

En una veta similar, Michael Lambek (1998: 111) ha descrito esta creatividad como poeisis, un término tomado de Aristóteles, que mueve el foco de atención desde los juicios sobre la veracidad o no del contenido narrativo hacia la comparación de la forma narrativa en sí. De manera más específica, poeisis se refiere al proceso de "producción creativa" desde el cual emergen todos los relatos del pasado (Lambek 1998: 111). Es por esta razón que Lambek (1998) prefiere el término "imaginación histórica" a "consciencia histórica", ya que mientras el primero reconoce el proceso creativo universal de involucramiento discursivo con el pasado, el segundo implica diferentes grados de proximidad con, y conciencia de, algunas de las narrativas maestras de la "realidad" o lo que "en realidad sucedió". 
Una comprensión estrictamente marxista de la consciencia histórica divide al mundo en dos tipos de personas: aquellos que conocen la "verdad" y aquellos que están engañados. Tal aproximación comete el error de no tomar en consideración la pluralidad de representaciones posibles del pasado, de las cuales cada una puede o no ser "verdad". No hay ninguna razón para que, por ejemplo, el foco sobre las vidas singulares central a muchas representaciones mapuche del pasado, sea más o menos objetivo que el análisis del cambio y movimiento social a gran escala común a mucha de la historiografía contemporánea. En ambos casos se crea una narrativa a través de un proceso de selección e inclusión. El asunto de la relativa "verdad" u "objetividad" de las diferentes formas de imaginación histórica es un tópico completamente diferente.

El "imaginarse" el pasado tan bien descrito por la perspectiva de White sobre la narrativa histórica, está íntimamente vinculado a otro tipo de "imaginación", la imaginación de una comunidad descrita por Anderson (1991). El autor expone como a través de un proceso creativo de inclusión y exclusión se construye una narrativa que bosqueja un límite alrededor de un grupo particular de gente, y como este límite es proyectado hacia el pasado. Este imaginar una comunidad puede ser tanto exclusivo y limitado como inclusivo y sin límites. El apartheid imaginando a Sudáfrica como una nación compuesta solamente por blancos europeos, excluyendo a los africanos negros, es un ejemplo de lo primero, mientras que la imaginación marxista de una comunidad global de base clasista es un ejemplo de lo segundo. Aun así, en cada caso la inclusión también implica exclusión y viceversa. Una forma particular de imaginación simultáneamente inclusiva y exclusiva es la "etnicidad", el imaginar una comunidad de gente que, según la perspectiva occidental, comparte prácticas culturales y lingüísticas, una historia y una sustancia común. Nuevamente quiero destacar que hablar de grupos étnicos como comunidades imaginadas no implica de ninguna manera que éstas no sean "reales", sino más bien, enfatiza el proceso particular a través del cual éstas se establecen. ${ }^{4}$

¿Cómo exploraremos estas narrativas, estos actos de poeisis? Un marco posible puede encontrarse en el trabajo del gran crítico ruso Mikhail Bakhtin, y particularmente en sus conceptos interrelacionados de "cronotopo" y "género". "La definición que Bakhtin entrega para cronotopo es la fusión de tiempo y espacio en el discurso. Esta definición es quizás algo imprecisa, ya que realmente él se centra mucho más en la representación de lo que llama la "imagen humana" y su relación con otros en la intersección de ejes temporales y espaciales. Es importante destacar que aunque Bakhtin está 
hablando sobre novelas, establece explícitamente que todas las formas de discurso implican de manera inevitable cronotopos particulares. Al trazar la evolución de los cronotopos, simultáneamente traza la evolución de distintas versiones de la "imagen humana" en los géneros literarios tempranos. Así, en el "tiempo de aventura" de la novela griega temprana los eventos de la trama toman lugar fuera del flujo de tiempo; los personajes centrales no envejecen, ni se desarrollan de ninguna forma. De la misma manera, la trama se ubica en tierras extrańas casi genéricas que carecen de particularidad o detalles. Debido a estar fuera del flujo de tiempo, la serie de eventos es completamente reversible. "El cronotopo de aventura se caracteriza así por una conexión técnica y abstracta entre espacio y tiempo, por reversibilidad de momentos en una secuencia temporal, y por su intercambiabilidad en el espacio" ${ }^{6}$ (Bakhtin 1981: 100). En la época de El Asno de Oro de Apuleyo, no obstante, el argumento se centraba en la metamorfosis del personaje principal, su desarrollo y maduración a través del tiempo. La propuesta central de Bakhtin acá es que cualquier forma de discurso implica inevitablemente una comprensión particular de las personas en una relación particular con la temporalidad.

El concepto de cronotopo está inextricablemente vinculado al de género. En efecto, como plantea Bakhtin (1981: 85) "es precisamente el cronotopo el que define al género". El género se refiere a la creación de un contexto en el cual puede emerger el significado. Todo discurso debe corresponder a un género si es significativo, pero esto no implica que los géneros estén de alguna manera fijos o sean formas estáticas. Más bien, nuevos contextos de significado están continuamente emergiendo y floreciendo a partir de los antiguos. El género es relevante para nuestro argumento en tanto establece los parámetros tácitos a través de los cuales se fundan los entendimientos particulares de la persona.

Es importante distinguir entre género en este sentido y género en el sentido de taxonomía metalingüística de los actos de discurso. Por ejemplo, mientras los mapuche reconocen los nütram y epew como categorías particulares de discurso, los géneros cronotópicos de singularidad o identidad que estaré describiendo podrían corresponder a ambos o a ninguno. Esto no implica negar que haya conexiones importantes entre las categorías nativas de discurso y las configuraciones particulares de persona y tiempo, sino más bien que éstas no están trazadas nítidamente entre ambas ${ }^{7}$. La relevancia de todo esto para mi propósito aquí es simple: que distintos géneros marcan diferentes configuraciones de tiempo y persona, y además, que cualquier configuración de tiempo implica una configuración de persona. El caso inverso también es cierto: cualquier configuración de persona implica 
una configuración de tiempo. La noción de género nos ayuda a comprender cómo configuraciones particulares se vuelven formas de representación y comprensión culturalmente arraigadas, punto que espero se aclare cuando me aboque a los ejemplos. Mi objetivo en este artículo es mostrar que la particular "historia" sobre la que se basa mucha de la retórica contemporánea del movimiento mapuche representa una configuración, un cronotopo si se quiere, del pasado y de la persona que es muy secundario en las representaciones del pasado que realizan los mapuche del mundo rural.

\section{Cronotopos de SINGUlaridad}

El primer cronotopo que quiero explorar es el del tiempo como compuesto por una serie de vidas únicas y singulares, configuración que se manifiesta de manera más evidente en las canciones personales, o ül. Las canciones caen en dos tipos que pueden ser etiquetados aproximadamente como seculares y religiosos ${ }^{8}$. Aquí me centro en las canciones seculares relativas a la experiencia personal, ya que son el tipo de ül ejecutado más comúnmente hoy en día en Piedra Alta e Isla Huapi, lugares donde he realizado mis investigaciones'. Lo que quiero sugerir es que estas canciones presentan una visión del pasado como si éste estuviera compuesto por series de personas necesariamente únicas y singulares, y no por miembros de grupos imperecederos. Las voces o puntos de vista desde los que emergen los $\ddot{u} l$ nunca son categorías genéricas de personas, ni tampoco constituyen representantes de grupos; en lugar de eso despliegan una visión singular del pasado en la cual la "historia" es la agregación de vidas individuales, nada más y nada menos.

Para justificar esta afirmación necesito explorar con mayor detalle la naturaleza real de estos $\ddot{u} l$. Los $\ddot{u} l$ emergen usualmente como canciones improvisadas en encuentros sociales informales. En el acto de improvisación, los compositores sólo cantan sobre sus propias experiencias y vidas; rara vez intentan enfatizar o representar las vidas de otros. Aunque la mayoría de las canciones nunca se repite, una respuesta entusiasta de parte de la audiencia puede inspirar al cantante a hacerlo en alguna otra ocasión. Estas canciones están íntimamente asociadas a su compositor inicial, y luego de su muerte continúan siendo cantadas por otros, los que a través de este proceso reviven, aunque sea temporalmente, los contornos de la vida del compositor, condensados en la canción. Regresaré a esta idea de habitación de las subjetividades pasadas a través de la canción más adelante. Por ahora es importante hacer notar que hay mucha más gente que canta canciones que la que las improvisa, por lo que la mayoría de las canciones que se cantan son las compuestas por otros sujetos ya fallecidos. Las canciones son siempre atribuidas a sus 
compositores iniciales, antes o después de ser interpretadas. En el caso de las canciones muy antiguas, cuyos compositores se desconocen, frecuentemente surgen debates acerca de la posible identidad del compositor inicial: ¿Dónde podría haber vivido?, ¿Qué experiencia lo motivó a cantar? El punto central aquí es que cada $\ddot{u} l$ es inextricable e inseparable de la subjetividad de su compositor, incluso si la identidad real del compositor se desconoce; entre los mapuche no puede haber actos genéricos de composición, como sucede en la tradición folclórica occidental.

¿De qué se tratan estas canciones? Sus temáticas se restringen casi exclusivamente a las relaciones entre afines en oposición a las que existen entre consanguíneos ${ }^{10}$. Las canciones desde la perspectiva de una mujer se refieren a la embustera naturaleza de los hombres, a la altamente constreñida relación con los yernos, al trauma que implica dejar la comunidad natal, y al estatus marital propio como algo básico para el cotilleo comunitario. De manera similar, las canciones de los hombres se refieren a la embustera naturaleza de las mujeres, a los celos de los hermanos de éstas, y a injustos rechazos amorosos. Ninguna de las canciones se refiere al amor filial, fraternal o hacia los padres, temas que son tan importantes en la tradición folclórica de Europa y otras partes del globo. Al mismo tiempo, aunque las canciones se refieren a relaciones de afinidad, casi siempre se dirigen a un interlocutor consanguíneo, usualmente un hermano.

En este sentido, la forma de las canciones se asemeja icónicamente a la forma de la persona mapuche. Porque, aunque cada persona emerge de su descendencia, küpal, de sus padres y sus abuelos, es a través del involucramiento en relaciones sociales con otros no relacionados a priori, por medio de la amistad o el matrimonio, como uno se convierte en una "persona verdadera”, en che (Course 2007, 2009b). Permítaseme explicar esto con mayor detalle: cada infante es producto del küpal, "descendencia”, de su madre y de su padre, descendencia que está vinculada a la de sus cuatro abuelos, descritos metafóricamente como meli folil, "cuatro raíces". El küpal es entendido como un componente "dado" de la persona: fijo, inmutable y permanente desde el momento de la concepción. Su influencia es visible en las características físicas de cada persona, en sus relaciones con espíritus, en su capacidad para cumplir ciertos roles sociales, y en su comportamiento moral. Aunque en teoría el küpal se vincula igualmente a la parentela matrilateral y patrilateral, usualmente es sólo la gente relacionada patrilateralmente la que es referida como kiñe küpal, "una descendencia". Ser de kiñe küpal implica una identidad compartida, una identidad que a su vez involucra una asistencia y solidaridad mutual. 
Aún así, para convertirse en una "persona verdadera", en un che, uno debe ir necesariamente más allá de estas relaciones de identidad dadas al momento de nacer, y demostrar la propia y autónoma volición a través de la creación de relaciones con no parientes, con afines potenciales, sean éstos amigos u amantes. Los saludos formales e informales, pentukun y chalintun, son reconocimientos explícitos a que el interlocutor es $c h e$.

En adición a este intercambio de palabras, es común el intercambio de bienes materiales, por medio de los cuales las relaciones con no parientes son creadas y mantenidas. Muchos de los eventos "comunales" de la vida mapuche, tales como el ritual de fertilidad del ngillatun y el deporte del palin, son mejor comprendidos si se los entiende como la agregación de numerosas relaciones diádicas de intercambio entre individuos. Para la mayoría de los mapuche el fin último de la vida social es el establecimiento de relaciones con no parientes, y la cooperación con individuos emparentados es a menudo vista simplemente como un medio para lograr este fin. Por ejemplo, hombres que comparten un küpal cooperarán en la organización de un juego de palin a objeto de que cada uno pueda, como individuo, proveer hospitalidad a sus amigos no emparentados.

El punto central aquí es que el concepto mapuche de la persona es claramente centrífugo, un movimiento constante hacia el exterior desde las relaciones consanguíneas dadas hacia la creación de relaciones con otros con los cuales no se está relacionado (Course 2005, 2007, 2008). Así, tal como la persona mapuche está enraizada en una relación de consanguinidad que sirve como un punto de anclaje para ingresar en relaciones de afinidad, de la misma forma la canción mapuche es un diálogo de afinidad incrustado en un diálogo de consanguinidad ${ }^{11}$. El $\ddot{u} l$ mapuche describe y se asemeja a este movimiento hacia el exterior, a este proceso de involucramiento con el otro; su referencia es tanto léxica como icónica. Un ejemplo de este rasgo en un ül típico puede observarse en la versión de Cornelio Painemilla de Wente Kawell Mallma, que está dirigida a un consanguíneo (ñaña, "hermana"), pero que trata de un afín potencial (wente kawell mallma, "jinete arrogante"):

Oh hermana querida, oh hermana, oh hermana / No te enamores de ese orgulloso jinete / Ese orgulloso jinete, quiere tener todo / No lo quieras, déjalo que siga su camino / No te enamores de ese orgulloso jinete, hermana querida / Ese orgulloso jinete, te pedirá todo / Incluso aunque ya tenga una esposa / "He perdido mi preciosa manta de montar bordada", dirá I "No tengo mi hermoso poncho", 
dirá toda clase de cosas / No te enamores de él, solo déjalo seguir su camino. ${ }^{12}$

¿Cómo es que estas canciones vienen a condensar una construcción tan singular de la "imagen humana", y además hacer que estas construcciones sean habitables por otros cantantes que toman prestadas las canciones en fechas posteriores? Quiero sugerir que es principalmente debido a su insistencia en los pronombres de primera persona, su entextualización, y su musicalidad, que los ül condensan la "voz" singular de su compositor inicial, y consecuentemente permiten que esta "voz" sea habitada por otros que se encuentran a gran lejanía temporal y espacial. De esta manera se plasma una representación particular del pasado, un cronotopo específico.

Como demostró Emile Benveniste en dos artículos clásicos sobre el tema (1971a, 1971b), es el empleo del pronombre de la primera persona lo que lía el lenguaje con el mundo. El pronombre de la primera persona Yo es el fundamento de toda referencia indicial, tanto para el tiempo como para el espacio. Además, es el pronombre de la primera persona el que permite justamente a la persona ingresar al lenguaje. Para Benveniste el lenguaje no es simplemente una representación de subjetividad, sino más bien se trata de que la subjetividad emerge a través del lenguaje. Cada vez que emplea el $Y o$, el hablante está tomando una posición distintiva vis-à-vis el mundo, y vis-àvis los otros. Pensemos en la famosa frase de Heidegger en referencia a que "el lenguaje es la casa del ser" (Heidegger 1971: 132). El tomar el Yo de otro es tomar, en mayor o menor medida, su posición en el mundo y su "voz".

Como he mencionado más arriba, todos los $\ddot{u} l$ mapuche están compuestos casi siempre en primera persona, y es esto lo que permite a las canciones capturar la experiencia de una persona en particular, y lo que también permite que los contornos de la vida del compositor sean capturados en la canción para ser revividos subjetivamente cuando ésta es cantada por otros posteriormente. De la misma manera, la estructura musical y poética relativamente fija del $\ddot{u} l$ permite que la "voz" del compositor inicial trascienda su contexto primario de emisión y se mueva a través del tiempo, proceso conocido como "entextualización" (Bauman y Briggs 1990, Silverstein y Urban 1996, Lempert y Perrino 2007). Los cantantes usualmente no intentan alterar ni improvisar sobre $\ddot{u} l$ de otros. Al contrario, tratan de mantener la forma y el contenido de la canción tal cual como la escucharon ${ }^{13}$. Esta fijeza de la canción condensa una visión singular de una persona única en un momento dado de tiempo, y luego un cronotopo particular. Así también, la musicalidad de la canción la distancia del discurso convencional en el que 
la voz del cantante sería sin problemas identificada como proveniente de su propia persona.

Hay, por cierto, mucho más que decir acerca de las canciones mapuche, por lo que esta resumida reseńa al respecto es apenas un breve esbozo ${ }^{14}$. Lo que quiero señalar para los propósitos de este artículo es que los $\ddot{u} l$ presentan una configuración particular de la persona y el pasado que se centra en la agencia individual en vez de hacerlo en categorías genéricas de personas, i.e. "hombre", "mujer" o "mapuche". Por tanto, el tiempo aparece como compuesto por una serie de vidas únicas. Otro punto a señalar es que los $\ddot{u} l$ mapuche son simultáneamente altamente específicos y muy generales. Específicos en el sentido de referirse a las minucias de la vida de una persona en particular, pero generales en el sentido de referirse a experiencias que escapan a cualquier temporalidad, como el amor, la traición y el aislamiento, que se encuentran removidas del flujo de eventos históricos. Luego, la imagen del pasado y la persona manifestadas en la canción, su cronotopo, presenta al pasado como compuesto por una serie de vidas singulares extraídas del paso de los grandes "eventos" históricos; vidas en que la pertenencia a grupos de gran tradición o la acción colectiva tienen muy poca importancia, si es que tienen alguna. El cantar un $\ddot{u} l$ permite tanto al cantante como a la audiencia involucrarse subjetivamente con esta versión del pasado.

Esta comprensión del pasado como una serie de vidas individuales únicas, que en la búsqueda de un mejor término denominaré "cronotopo de singularidad", no se encuentra limitada al ül. De hecho es más común encontrarla en las miríadas de historias y comentarios relativos a individuos en particular, tanto vivos como fallecidos. La mayoría de estas historias, mas no todas, podrían ser clasificadas como nütram o "historias verdaderas". Éstas difieren de los $\ddot{u} l$ en que son contadas en tercera persona y no hay ninguna vinculación al habitar las subjetividades antes descrito. Sin embargo, los nütram presentan una comprensión similar de la exclusividad de las vidas individuales que se encuentran fuera del mentado flujo de "eventos". Estas historias pueden ser humorísticas o trágicas, moralistas o didácticas.

Tomemos, por ejemplo, una historia que me contó Sergio Painemilla sobre Lonkomila, un anciano ciego que arribó al lago Budi, en compañía de su joven esposa, durante los que podrían ser, estimativamente, los últimos años del siglo XIX. Lonkomilla golpeaba y maltrataba a su esposa hasta que ella comenzó a desesperarse por dejarlo. Pero en su astucia, Lonkomilla siempre se aseguraba de esconder el único bote a remos que había, que era además la única forma de acceso a la isla. Sin embargo, su crueldad era tan 
grande que la mujer finalmente se arrojó caminando por el lago, prefiriendo la muerte a una vida junto a él. Con mucha suerte para ella, el nivel del agua se encontraba muy bajo, por lo que pudo llegar a la orilla y retornar donde su parentela. Esta historia se centra en la particularidad y singularidad de un individuo en específico, Lonkomilla, con la exclusión de un contexto histórico particular; Lonkomilla y su esposa eran refugiados que venían desde las cercanías de Pitrufquen, escapando del derramamiento de sangre provocado por el ejército chileno. Incluso las historias que hablan sobre individuos aún vivos se refieren a su singularidad y particularidad en vez de cualquier membrecía a un agregado social, sea éste un grupo de parentesco o "étnico".

\section{Cronotopos De identidad}

Hasta el momento me he centrado en un cronotopo de singularidad que presenta al pasado como la simple acumulación de vidas individuales, pero podría inducir a errores el dejar la descripción de éste hasta ahí. Debemos considerar que el término "mapuche" existe, en efecto, como una categoría notable en las narrativas sobre el pasado, y es a este cronotopo de identidad al que me abocaré ahora.

La comprensión de "mapuche" a la que me estoy refiriendo aquí no está confinada a ningún género en particular, sino que aparece en una amplia diversidad de discursos cotidianos, desde cuentos hasta canciones. Lo que quiero señalar es que para mucha gente rural, especialmente para la generación mayor, el término "mapuche" no se refiere a un grupo delimitado de personas que comparte una historia y ubicación geográfica común (en otras palabras, a una identidad "étnica"), sino más bien a una categoría universal de personas en un tipo particular de relación estructural de producción y poder con otros.

Mi objetivo en este relato no es, ciertamente, entregar una definición absoluta sobre lo que significa mapuche, ni tampoco un listado consistente de criterios en base a los cuales se atribuya la identidad "mapuche" en el discurso rural. Lo que quiero demostrar es que el significado de "mapuche" es siempre relativo, y que emerge a partir del contexto particular en que es empleado el término. Es por esta razón que existen numerosas contradicciones en que la gente rural atribuye la identidad mapuche. Un ejemplo conocido tiene que ver con la insistencia de mucha gente del campo en referencia a que todos los mapuche hablan mapudungun, y a que es, por tanto, su capacidad lingüística un rasgo definitorio de su mapuchidad, aunque en otros contex- 
tos la misma gente pueda reconocer a no hablantes de mapudungun como mapuche. Las contradicciones implicadas en la atribución de identidad no son exclusivas a la sociedad mapuche, y de hecho parece ser un aspecto muy común, e incluso tal vez necesario, de la adscripción identitaria en todo el mundo. El sociólogo Gerd Bauman (1996), a partir de su propia investigación sobre las adscripciones identitarias étnicas en Inglaterra, ha descrito esta co-existencia de comprensiones contradictorias de la identidad como "competencia discursiva dual".

Las ideas acerca de qué significa "mapuche" se revelan con mayor claridad en lo que podríamos denominar la "etno-antropología" mapuche, es decir, en las preguntas que los propios mapuche me hicieron con respecto a la vida social en Inglaterra. Frecuentemente se me consultó cómo eran los mapuche en Inglaterra, si hablaban mapudungun, si eran ricos o pobres, cómo era la calidad de sus tierras, etc. Estas preguntas no apuntaban al pequeño grupo de mapuche exiliados a Inglaterra durante la dictadura, sino más bien a los supuestos habitantes nativos del país. También me hicieron preguntas similares sobre otras partes del mundo: ¿eran los negros de Brasil, los afrobrasileños, los mapuche brasileños? Varias veces escuché comentarios de la gente en referencia a que los indígenas amazónicos que veían en televisión no podían ser mapuche, debido a que vivían en "tribus" y no en hogares separados (como lo hacen los mapuche rurales). Todas estas preguntas y comentarios emergían de una afirmación frecuente: "todos los países del mundo tienen sus propios mapuche". Luego, ¿qué significa mapuche en este contexto? Pienso que se refiere principalmente a dos cosas: al modo de subsistencia y a la relación estructural con los no mapuche, denominados winka.

Mapuche significa literalmente "gente de la tierra", y me parece pertinente detenerme aquí para reflexionar acerca de esta etimología particular, pues creo que lo notable del término yace en su referencia a una manera específica de vivir, encarnada por una producción agrícola de pequeña escala. En una influyente propuesta, Guillaume Boccara $(1998,1999)$ ha sostenido que la relativamente reciente aparición del etnónimo "mapuche" evidencia un proceso de etnogénesis ${ }^{15}$. El término no aparece en el registro documental sino hasta el siglo XVIII, y Boccara explica que anteriormente los indígenas de la zona centro-sur de Chile se autodenominaban reche, "gente pura”, aunque sólo cuenta con un documento para sostener su hipótesis (cf. Dillehay 2007).

Lo que quiero señalar es que, de manera opuesta a las insistencias de Boccara, la emergencia del término "mapuche" no se refiere necesariamente 
a la emergencia de un grupo étnico discreto, sino que puede referirse, igual y sencillamente, a la aparición de un nuevo modo de vida, aquél de los asentamientos agrícolas que vino a reemplazar al modo anterior de subsistencia, basado en una combinación de horticultura, caza y recolección. La relevancia de una identidad basada en un modo de vida radica en que no se refiere necesariamente a una historia particular compartida, como sucede con la identidad étnica. De ahí, la afirmación "deben haber mapuche en todos los países" es completamente correcta, si entendemos mapuche como un término aproximadamente equivalente a campesino. No obstante, ciertamente una afirmación como ésta debe ser entendida siempre como relativa: en algunos casos los mapuche son opuestos a los no mapuche como los campesinos lo son con los latifundistas. En otros contextos, ciertamente, la gente reconoce que muchos no mapuche también viven como campesinos. La importancia del modo de producción en la definición de la identidad mapuche parece tocarse con una distinción más amplia entre mapuche y winka, cuestión que trataré a continuación.

En muchos contextos el término "mapuche" se refiere a una relación particular entre éstos y los no mapuche o winka, concepto de origen etimológico poco claro. Lo que sí es claro es que ser mapuche es opuesto a ser winka, y que los dos términos son empleados retóricamente para abarcar una serie de oposiciones (cf. González 2007) ${ }^{16}$. Los mapuche son pobres, los winka ricos, los mapuche honestos, los winka deshonestos, y así sucesivamente. La identidad mapuche incrustada en la producción agrícola no está limitada a la ocupación de un lugar específico o al compartir una historia, y lo mismo sucede con la identidad formada en oposición con los winka: la distinción mapuche/winka es universal. Esta comprensión de lo que significa mapuche de cierta manera se asemeja a un entendimiento casi marxista de identidad de clase, en que la humanidad está universalmente dividida en clases distintas envueltas en una pugna antagónica por los recursos. Pero tal ecuación sería demasiado simplista. A menudo las adscripciones de mapuchidad implican de hecho cierto grado de esencialismo cultural; por ejemplo, como se ha descrito más arriba, es común la afirmación relativa a que los "mapuche" hablan mapudungun universalmente y que viven en asentamientos dispersos y no en aldeas. De esta manera, aunque las definiciones de "mapuche" a menudo giran en torno a una oposición relativa con los winka, el contenido real de lo que significa "mapuche" y winka puede variar de acuerdo al contexto.

Existen muchos paralelos entre estas comprensiones mapuche rurales de la identidad y aquellas descritas por Rosengren (2003) para los Matsiguenka de la Amazonía Peruana. Empleando las metáforas de Deleuze de 
"rizoma” y "raíz primaria", Rosengren opone los conceptos Matsiguenka de identidad que enfatizan la inclusividad, el proceso y el contacto cara a cara, con los conceptos de identidad introducidos por las ONG y los Matsiguenka educados formalmente, que enfatizan la exclusión, la esencia y una consecuente solidaridad "étnica". Mientras que esta última comprensión de la identidad como una raíz primaria enfatiza una conexión particular con una trayectoria histórica singular, la primera metáfora del rizoma pone énfasis en la pluralidad de modos de establecer relaciones de identidad. Estas dos formas de comprender la identidad no son de manera necesaria mutuamente excluyentes, y ambas pueden aparecer dependiendo del contexto. De este modo, la gente puede emplear acertadamente ideas contradictorias relativas a la identidad, enfatizando algunas veces la identidad en su carácter fijo, permanente y esencial, mientras en otras haciendo referencia al proceso y al contexto. El caso mapuche no es diferente: como hemos visto más arriba, muchos mapuche rurales afirman que todos los mapuche hablan mapudungun y lucen como mapuche. No obstante, los mismos individuos no tienen problemas en atribuir la identidad mapuche a gente que no habla mapudungun ni luce como mapuche, sobre la base de tener uno o más padres mapuche. El punto central aquí no es tratar de desenredar estas contradicciones aparentes, sino reconocer que en muchas instancias la identidad mapuche se trata de una cosa distinta a pertenecer a un grupo "étnico" concebido de manera excluyente. Para muchos mapuche rurales, una comunidad específicamente mapuche con un pasado e intereses comunes aún debe ser imaginada. El alcance de su identidad social compartida está, como para los Matsiguenka, limitado a aquellos con quienes pueden tener un contacto cara a cara.

Ahora bien, ciertamente no deseo dar la impresión de que una conceptualización étnica de la identidad mapuche extendida hacia el pasado se encuentra completamente ausente del discurso rural. Hasta cierto punto la oposición que aquí se hace entre los contextos rural y urbano es engañosa: la mayoría de los adultos ha pasado al menos algún tiempo en centro urbanos como Temuco y Santiago, y un número creciente de jóvenes recibe educación secundaria y universitaria en Temuco. Adicionalmente, una versión "étnica" de la identidad mapuche es parte de los contenidos obligatorios entregados en las escuelas primarias. En todos estos contextos la gente guarda la visión de los mapuche como un grupo étnico unificado y delimitado, con una historia e intereses comunes; aunque la relevancia de esta visión es a menudo cuestionada. Frecuentemente me vi sorprendido por el poco interés o preocupación que mostraba la gente de las comunidades con respecto a los diferentes conflictos existentes en la Araucanía, o incluso con referencia 
a la construcción de la central hidroeléctrica de Ralco. "¿Qué tengo que ver yo con esa gente allá?", me dijo una vez una mujer. Para muchos mapuche de alrededor del Budi, las luchas de otras comunidades en otras partes del sur aparecen como muy lejanas. Para ellos ser mapuche no implica ningún lazo o interés común. Puede relacionarse con una forma común de vida o una experiencia común de opresión, pero esto no se convierte en una identidad "étnica" enraizada en una historia compartida. El pasado es representado primariamente como la acumulación de individuos únicos y singulares, y no como la acción de distintos grupos delimitados. Estas narrativas de las vidas pasadas enfatizan la acción individual y la responsabilidad en sus implicaciones morales y políticas, en vez de enfatizar la acción política sobre la base de una solidaridad étnica.

\section{Conclusión}

Mi objetivo en este ensayo ha sido explorar las maneras en que los discursos sobre el pasado de los mapuche rurales alrededor del Lago Budi ofrecen una multiplicidad de configuraciones sobre el tiempo y la persona, las que no necesariamente corresponden a la versión "étnica" de la identidad mapuche que depende de un cronotopo que está frecuentemente ausente o es secundario en el discurso antes mencionado. Esta incongruencia en las comprensiones sobre el tiempo y la persona es quizás una explicación parcial para la marcada ausencia de participación y entusiasmo entre muchos mapuche rurales con respecto al movimiento mapuche. Ciertamente no quiero sugerir que esta situación sea particular a los mapuche; en muchos otros casos existe una disyunción entre las historias locales y las grandes narrativas de la historia "oficial", empleadas con fines políticos. Tampoco quiero sugerir que las narrativas rurales sobre el pasado sean más auténticamente "mapuche" que otras, ya que las comprensiones del pasado son en sí objetos históricos susceptibles de cambio y transformación. Simplemente quiero destacar la diversidad de entendimientos existentes sobre el pasado en la vida rural mapuche, y sugerir que éstos merecen consideración por propio derecho.

Es inevitable que sobre el movimiento mapuche, y otros movimientos indígenas de las Américas, debido a su posicionamiento entre ontologías políticas múltiples, emerjan contradicciones o incongruencias ${ }^{17}$. Mas ofrecer críticas como las expresadas aquí claramente no es suficiente. El pueblo mapuche vive en un Estado notoriamente contrario a sus demandas, al punto de negarse durante muchos ańos a la ratificación del convenio 169 de la OIT, que les daba espacio a los mapuche en la Constitución. Estos rechazos legales 
y políticos han tenido profundos y crecientes efectos negativos en la vida mapuche urbana y rural, los que continúan hasta el día de hoy. La crítica literaria ofrece poco consuelo a quien es acribillado por la espalda mientras huía de la policía chilena. En tal contexto, el imaginar una identidad "étnica” mapuche quizás no es sólo necesario, sino inevitable. Así, supongo que lo que quiero proponer como conclusión es que un sentido de pertenencia a una identidad "étnica" mapuche en las comunidades rurales es algo que debe ser creado y no asumido. Y esta creación no debe basarse en la supuesta "ignorancia" de la población rural, o en su supuesta carencia de memoria histórica, sino más bien en tener conciencia y respetar la sutileza y alcance de su imaginativo involucramiento con el pasado.

Agradecimientos: La investigación que dio pie a este artículo fue financiada por el Economic and Social Research Council y la British Academy; quiero agradecer a ambas instituciones por su apoyo. Estoy especialmente agradecido de Ana Mariella Bacigalupo, Rolf Foerster, Marcelo González, André Menard y Claudia Robles por sus comentarios y sugerencias a las versiones preliminares de este trabajo. Tambien agradezco a los evaluadores anónimos por sus sugerencias y comentarios. Mi mayor deuda la tengo con las numerosas personas de Piedra Alta y Huapi que han compartido conmigo sus vidas, historias y canciones durante muchos años. Este artículo fue traducido por Marcelo González Gálvez.

\section{Notas}

1 Las estrategias reales empleadas por organizaciones específicas dentro del movimiento mapuche a menudo intentan poner sus asuntos en términos familiares a la realidad rural. Sin embargo, estos términos con frecuencia se fundamentan en una comprensión objetivada y reificada de lo que es la cultura mapuche rural. Tomemos, por ejemplo, la insistencia en las "autoridades tradicionales" o la "organización territorial", que son conceptos ampliamente utilizados que a menudo distorsionan y oscurecen la fluidez de la organización política y territorial de la sociedad mapuche rural (Course 2009b).

2 Tómese, por ejemplo, el famoso malentendido sobre la distinción de Lévi-Strauss (1966) entre sociedades "calientes" y "frías". Mientras que las sociedades "calientes", como las europeas, representan el pasado como una progresión continua basada en un cambio acumulativo, las sociedades frías, como las de los indígenas de Sudamérica, buscan enfatizar la continuidad y negar la aparición del cambio. Aunque algunos críticos han acusado a Lévi-Strauss de sostener que los pueblos indígenas no poseen "historia" (Hill 1988), mi comprensión de su propuesta va simplemente por apuntar a que el pasado puede ser representado de numerosas maneras diferentes, entre las cuales la "historia” es sólo una más (véase Gow 2001) para una discusión detallada sobre estos asuntos). Las implicancias políticas de esta disyunción entre las representaciones amerindias y europeas del pasado es discutida por Lévi-Strauss (1996) en el magnífico capítulo final de The History of Lynx [Historia de Lince, 1992, Anagrama, Barcelona].

3 Debo aclarar que mi empleo de una distinción entre urbano y rural es primariamente retórica; no describe adecuadamente la complejidad de la vida mapuche contemporánea, en la que muchas personas son simultáneamente rurales y urbanas. Además, mi comprensión de la vida mapuche rural se basa en los ańos durante los que viví en comunidades mapuche en la zona del Lago Budi, área que de muchas maneras es atípica en comparación con la situación mapuche rural general. 
4 Hay, por cierto, bastante literatura sobre el proceso de "etnogénesis" en Latinoamérica, que busca describir cómo los grupos étnicos contemporáneos emergen como unidades delimitadas diferentes debido al proceso de colonialismo histórico (Hill 1996). La situación colonial lleva a una objetivación y fijación de las identidades que antes habían sido fluidas y procesuales. Un argumento similar sostiene Boccara (1998, 1999), para los mapuche, sugiriendo que una identidad mapuche colectiva sólo emergió durante el siglo XIX.

5 La idea de cronotopo es desarrollada en el ensayo Forms of Time and of the Chronotope in the Novel (Bakhtin 1981), mientras que la noción de género es descrita en The Problem of Speech Genres (Bakhtin 1986).

6 "The adventure chronotope is thus characterized by a technical, abstract connection between space and time, by the reversibility of moments in a temporal sequence, and by their interchangeability in space".

7 Por ejemplo, es tentadora la idea de traducir epew como "mito" y nütram como "historia", pero esto sería una severa sobre simplificación, ya que tanto los epew como los nütram utilizan varios cronotopos.

8 Véase Painequeo (2000) y Course (2009a) para una profundización sobre los ül.

9 La investigación sobre la que se basa este artículo fue realizada en un total de 30 meses entre los años 20012003, 2005 y 2007, tiempo durante el cual viví en Piedra Alta, lugar ubicado entre el lago Budi y el océano Pacífico, en la comuna de Puerto Saavedra, Novena Región.

10 La distinción que realizo entre relaciones de "afinidad" y "consanguíneas" se basa en el concepto mapuche de küpal, "descendencia”. Aunque tanto los parientes matrilaterales como los patrilaterales comparten küpal, son sólo estos últimos los que se dice que pertenecen a kiñe küpal, “un küpal” (Course 2005, 2007, 2009b).

${ }^{11} \mathrm{La}$ afinidad de la que hablo aquí no es solamente la afinidad real creada a través del matrimonio, sino todo el reino simbólico de alteridad que Viveiros de Castro (2001) ha descrito como "afinidad potencial".

12 Naña anay, Naña anay, Naña anay / Duamfelay wente kawell mallma, wente kawell mallma, ñaña anay, ñaña anay / Wente kawell mallma trokompituy wente kawell mallma / Duamfelay kalikalimiawpe wente kawell mallma / Duamfelay duamfelay wente kawell mallma ñaña anay / Wente kawell mallma itrokomngapikeli / Ranginga ñi ngen tañi kure / "Nien ñami ñimin lama" pikeynga wente kawell mallma / "Nielanga nielanga kume makuin" itrokomnga pikey / Duamfelay duamfelay kalikalimiawpe.

13 Con esto no estamos sosteniendo que las canciones no cambien con el tiempo, ya que ciertamente lo hacen. Lo que sí proponemos es que los cantantes se auto perciben como repitiendo una forma relativamente fija, aún cuando en la práctica se hallen modificándola.

14 Una versión más detallada de mi propuesta sobre el ül y la subjetividad se encuentra en Course (2009a).

15 Véase Zavala (2008) para una perspectiva distinta.

16 Cabe mencionar que la auto atribución de autoctonía o estatus primario para los mapuche es a menudo secundaria o periférica en las ideas de la gente acerca de la distinción entre mapuche y winka. Los winka son a menudo descritos no necesariamente como gente de un lugar distinto, sino como personas que han estado siempre presentes en la vida mapuche de una u otra forma. Este es otro ámbito en el que las ideas rurales sobre la autoctonía, y la historia, está en contradicción con las de otros lugares.

17 Véase Foerster y Vergara (2003) para una discusión más profunda sobre el tema.

\section{BiBLIOGRAFÍA}

Anderson, B. 1991. Imagined Communities: reflections on the origin and spread of nationalism. Verso, London.

Bakhtin, M. 1981. The Dialogic Imagination: essays by M.M. Bakhtin. University of Texas Press, Austin.

Bakhtin, M. 1986. Speech Genres and Other Late Essays. University of Texas Press, Austin.

Baumann, G. 1996. Contesting Culture: Discourses of Identity in Multiethnic London. Cambridge University Press, Cambridge. 
Bauman, R. y C. Briggs. 1990. "Poetics and performance as critical perspectives on language and social life". Annual Review of Anthropology 19: 59-88.

Benveniste, E. 1971a. "The Nature of Pronouns". En Problems in General Linguistics, pp: 217-223. University of Miami Press, Miami.

Benveniste, E. 1971b. "Subjectivity in Language". En Problems in General Linguistics, pp: 223-230. University of Miami Press, Miami.

Boccara, G. 1998. Guerre et ethnogenèse Mapuche dans le Chili colonial: l'invention du soi. L'Harmattan, Paris.

Boccara, G. 1999. "Etnogénesis mapuche: resistencia y restructuración entre los indígenas del centro-sur de Chile (siglos XVI-XVIII)". Hispanic American Historical Review 79: 425-461.

Course, M. 2005. "Borges, the Mapuche, and the Mother's Brother's Son". Cambridge Anthropology 25: 11-30.

Course, M. 2007. "Death, Biography, and the Mapuche Person". Ethnos 72: 77-101.

Course, M. 2009a. "Why Mapuche Sing". Journal of the Royal Anthropological Institute 15: 295-313.

Course, M. 2009b. Mapuche Ni Mongen: individuo y sociedad en la vida mapuche rural. Editorial Universidad de la Frontera, Temuco.

Dillehay, T. 2007. Monuments, Empires, and Resistance: The Araucanian Polity and RitualNarratives. Cambridge University Press, Cambridge.

Foerster, R. y J. Vergara. 2003. "Etnia y nación en la lucha por el reconocimiento. Los mapuches en la sociedad chilena". En Mapuches y Aymaras: El Debate en Torno al Reconocimiento y Los Derechos Ciudadanos, editado por H. Gunderman, R. Foerster, y J. Vergara, pp: 105-178. Editores RIL, Santiago.

González, M. 2007. Entre el Don y el Bien Limitado: Hacia el Desentrañamiento del Ethos Segmental. Poder social entre los mapuches de Huentelolén, Provincia de Arauco. Memoria para optar al título de Antropólogo Social, Departamento de Antropología, Facultad de Ciencias Sociales, Universidad de Chile. 
Gow, P. 2001. An Amazonian Myth and Its History. Oxford University Press, Oxford.

Heidegger, M. 1971. Poetry, Language, Thought. Harper \& Row, New York.

Hill, J. (ed.) 1988. Rethinking History and Myth: indigenous South American perspectives on the past. University of Illinois Press, Urbana.

Hill, J. (ed.) 1996. History, Power and Identity: Ethnogenesis in the Americas, 1492-1992. University of Iowa Press, Iowa.

Lambek, M. 1998. "The Sakalava Poiesis of History: realizing the past through spirit possession in Madagascar". American Ethnologist 25 (2): 106-127.

Lempert, M. y S. Perrino. 2007. "Entextualization and the Ends of Temporality". Language and Communication 27: 205-211.

Lévi-Strauss, C. 1966. The Savage Mind. Weidenfeld \& Nicholson, Londres.

Lévi-Strauss, C. 1996. The Story of Lynx. University of Chicago Press, Chicago.

Marimán, P., S. Caniuqueo, J. Millalén y R. Levil, 2006. ;...Escucha, winka...! Cuatro ensayos de Historia Nacional Mapuche y un epilogo sobre el futuro. Ediciones LOM, Santiago.

Painequeo, H. 2000. La Oralidad en el Canto Mapuche. CIESAS, México, D.F.

Rosengren, D. 2003. "The Collective Self and the Ethnopolitical Movement: 'rhizomes' and 'taproots' in the Amazon". Identities 10: 221-240.

Silverstein, M. y G. Urban. (eds). 1996. Natural Histories of Discourse. University of Chicago Press, Chicago.

Viveiros de Castro, E. 2001. Gut feelings about Amazonia: potential affinity and the construction of sociality. En Beyond the visible and the material: the Amerindianization of society in the work of Peter Riviere, L. editado por Rival y N. Whitehead (eds.), pp: 19-43. Oxford University Press, Oxford. 
White, H. 1973. Metahistory: The Historical Imagination in 19th- Century Europe. Johns Hopkins Press, Baltimore.

White, H. 1987. The Content of the Form: Narrative Discourse and Historical Representation. Johns Hopkins Press, Baltimore.

Zavala, J. M. 2008. Los Mapuches del Siglo XVIII: Dinámica Interétnica y Estrategias de Resistencia. Editorial Universidad Bolivariana, Santiago. 